\title{
Evolutionary Chance Mutation: A Defense of the Modern Synthesis' Consensus View
}

\author{
Francesca Merlin ${ }^{\S}$
}

\begin{abstract}
One central tenet of the Modern Evolutionary Synthesis (1930s-1950s), and the consensus view among biologists until now, is that all genetic mutations occur by "chance" or at "random" with respect to adaptation. However, the discovery of some molecular mechanisms enhancing mutation rate in response to environmental conditions has given rise to discussions among biologists, historians and philosophers of biology about the "chance" vs "directed" character of mutations (1980s-2000s). In fact, some argue that mutations due to a particular kind of mutator mechanisms challenge the Modern Synthesis because they are produced when and where needed by the organisms concerned. This paper provides a defense of the Modern Synthesis' consensus view about the chance nature of all genetic mutations by reacting to Jablonka and Lamb's analysis of genetic mutations (2005) and the explicit Lamarckian flavor of their arguments. I argue that biologists can continue to talk about chance mutations according to what I call and define as the notion of "evolutionary chance," which I claim is the Modern Synthesis' consensus view and a reformulation of Darwin's most influential idea of "chance" variation. Advances in molecular genetics are therefore significant but not revolutionary with respect to the Modern Synthesis' paradigm.
\end{abstract}

\section{KEYWORDS \\ Biased chance $\bullet$ Chance $\bullet$ Darwin $\bullet$ Directed mutation $\bullet$ Lamarckism $\bullet$ \\ Modern Synthesis $\bullet$ Mutator mechanism $\bullet$ Random mutation}

\section{Introduction}

Biologists usually agree that all genetic mutations occur by "chance" or at "random" with respect to adaptation. The claim dates back to Darwin's conception of "spontaneous," "accidental" or "chance" variation (Darwin 1859, 1868; Darwin and Seward 1903). The Modern Synthesis later redefined Darwin's idea as rooted in the phenomenon of genetic mutation following a long period of controversy over the "chance" vs "directed" character of variation.

The main purpose of this paper is to defend the "chance" character of genetic mutations, which I claim is a Darwinian tenet and part of the Modern Synthesis' consensus view, against recent challenges, especially those advanced by Jablonka and Lamb $(1995,2005)$. During the last thirty years, experimental research in molecular genetics, in particular on microorganisms, has shown that certain molecular mechanisms - the socalled "mutator mechanisms" - can regulate mutation rates (increasing or decreasing them) in response to certain selective forces. Because of this causal connection between mutation rates and selective substrates, Jablonka and Lamb, along with other biologists, historians and philosophers of biology (Shapiro 1999, 2005;

\footnotetext{
§ Department of Philosophy, University of Montréal, 2910 Edouard Montpetit Blvd., Québec, H3T 1G7 Canada E-mail: francesca.merlin@gmail.com
}

Received 24 May 2010; Revised 28 July 2010; Accepted 12 August 2010 
Wright et al. 1999, 2000; Sternberg 2002; Keller 2000), have questioned the Modern Synthesis' claim that all genetic mutations occur by "chance" or at "random."

The present paper refines Millstein's conceptual and empirical analysis of the old distinction between "random" and "directed" genetic mutation (Millstein 1997) and provides a definitive argument against Jablonka and Lamb's influential idea that mutations brought about by some kinds of mutator mechanisms are "non random" because they are produced by partially Lamarckian processes when and where needed. As I will argue, a return to Lamarck's ideas, like the one advocated by Jablonka and Lamb, is an unnecessary conceptual and empirical requirement for explaining mutations due to these mechanisms.

I will argue instead that all genetic mutations, including those due to mutator mechanisms, can be accounted for by the Modern Synthesis' consensus view since they are not specifically caused in an (exclusively) adaptive way by a physico-chemical process in response to environmental conditions (what I call "evolutionary chance" or "non directed" mutations). In so doing, I will draw upon the empirical evidence available within the consensual theoretical framework on genetic mutations and pay particular attention to some relevant advances in molecular genetics. ${ }^{2} \mathrm{I}$ will also underline the importance of certain distinctions well known in biology but seldom used to defend the Modern Synthesis' consensus on genetic mutations. This paper focuses exclusively on mutations, i.e., the sources of genetic hereditary variation, and will not provide any analysis of recent research in epigenetic variation and inheritance more broadly construed.

The paper is divided into two parts. The first part (Sections 2-5) discusses the Modern Synthesis' view that all genetic mutations are a matter of "chance." I will begin by reviewing different formulations of this idea and then move on to show that the Modern Synthesis' consensus view corresponds to what I call "evolutionary chance mutation." The second part (Sections 6-11) pertains to Jablonka and Lamb's challenge to the Modern Synthesis' consensus view. I will examine the notion of "evolutionary chance mutation" in light of the most controversial kind of mutator mechanism, and will show why Jablonka and Lamb's distinction between "random" and "non random" mutations is both conceptually and empirically inappropriate. These two parts will work to show that recent advances in molecular genetics, which may seem to transform our understanding of genetic mutation, do not, in fact, represent a revolutionary (Lamarckian) departure from the Modern Synthesis's idea of "evolutionary chance mutation." Such a conclusion may be of interest to philosophers because of the conceptual clarification it provides. It is also of concern to practicing evolutionary biologists insofar as showing that recent findings about genetic mutations are less revolutionary than some claim liberates their research from its purported conflict with the Modern Synthesis consensus view.

\section{Ghance Mutation: A Variety of Formulations}

According to the Modern Synthesis, there are two sources of genetic variation: mutation and recombination. Ever since the discovery of the physico-chemical structure of the genetic hereditary material (the DNA) in the 1950s, the term "mutation" has referred to all types of changes in DNA nucleotide sequence, except those due to the recombination of fragments of genetic material exchanged between paired DNA strands. Both sources of genetic variation are considered unpredictable phenomena as to the time they occur, the genes they affect, and the individual organisms concerned (Dobzhansky 1951 [1937], 45; Simpson 1984 [1944], 86; Dobzhansky 1970, 92). In particular, a central tenet of the Modern Synthesis claims that genetic mutations occur by "chance" or at "random" with respect to adaptation, that is, with respect to the adaptive needs of the organisms concerned and the population to which they belong.

The Modern Synthesis' canonical texts ${ }^{3}$ allow us to identify a variety of more or less ambiguous formulations used by biologists to express the idea of "chance mutation." Indeed, sometimes the meaning given to the terms "chance," "random," and other expressions, none of which are ever explicitly defined, are so vague that they can be interpreted differently and may seem to contradict empirical evidence about genetic variation and its possible causes. These formulations can be divided into three groups:

1. The first group brings together formulations which explicitly evoke the notion of chance via terms like "random" and "chance" itself. 
"Random from the point of view of adaptation and functional integration" (Simpson 1984 [1944], 55-56); "Random with respect to the direction of adaptation" (Stebbins 1966, 35); "A random process with respect to the adaptive needs of the species" (Dobzhansky 1970, 65).

2. The second group rarely uses the terms "chance" and "random," but nevertheless employs expressions and ideas that imply the notion of chance to characterize genetic mutations (e.g., the ideas of absence of correlation and of independence).

"No correlation has been found between external conditions and direction of mutation" (Wright 1931, 142); "The directions of the changes produced by them [mutations] appear to be unrelated either to the direction of the evolutionary change to be observed in the type, or the adaptive or functional needs of the organism" (Huxley 1948 [1942], 54); "Mutations are random changes because they occur independently of whether they are beneficial or harmful, and therefore they are a disordering process" (Dobzhansky et al. 1977, 66).

3. Finally, the third group contains formulations that define the chance character of genetic mutations in the negative. These formulations oppose the Modern Synthesis' conception of genetic mutations to Lamarckian theories of evolution and, more specifically, to Lamarck's conception of hereditary variation and adaptation.

"The nature of the mutations observed is not compatible with the view that evolution is directed by their means" (Fisher 1999 [1930], 20); "An ideal situation would be if the organism were to respond to the challenge of the changing environment by producing only beneficial mutations where and when needed. But nature has not been kind enough to endow creations with such a providential ability" (Dobzhansky 1951 [1937], 51, 74); "On the other hand, the term 'randomness' as applied to mutation often refers to the lack of correspondence of phenotypic effect with the stimulus and with the actual or the adaptive direction of evolution. Heat-induced mutations do not produce phenotypic change related to heat tolerance" (Simpson 1953, 87).

All three groups retain the essential Darwinian notion of "spontaneous," "accidental," or "chance" variation with respect to adaptation ${ }^{4}$ and define the chance character of genetic mutations in evolutionary terms. They explicitly refer to the relationship between the occurrence of mutations and their adaptive value for the organisms concerned and their species. However, they do not unambiguously allow us to understand what the biologists of the Modern Synthesis meant by "chance" or "random." In fact, the meaning of these terms is not explicitly defined at all. For this reason, all these formulations can be easily misinterpreted.

In the first group of formulations, the meaning of the term "random," which biologists use more often than the term "chance," is not defined and is therefore open to different interpretations, some of them improper. The expressions used by the second group seem to be clearer since they replace the term "random" with more meaningful ones, such as "independence" or "absence of correlation". But these formulations nevertheless remain ambiguous since they neither state which of the elements is independent nor the nature of its independence - statistical or causal. ${ }^{5}$ The formulations of the third group tell us more since they clearly express how biologists of the Modern Synthesis did not conceive genetic mutations, i.e., in opposition to a Lamarckian account of the origin and character of hereditary variation. For this reason, the third group is the most useful in providing a good definition of the notion of chance mutation, and for understanding whether the discovery of molecular mechanisms regulating mutation rates challenges this notion.

Having distinguished three ways of expressing the idea of "chance mutation" and underlined their ambiguity, let us now review the views held by biologists of the Modern Synthesis on genetic mutations. ${ }^{6}$ This review will allow us to consider whether the advances in molecular genetics over the last thirty years, in particular the discovery of molecular mutator mechanisms, represent a real challenge to the Modern Synthesis consensus view (i.e., the "chance" character of all genetic mutations). 


\section{Mutational Bias at the Time of the Modern Synthesis}

The first step in identifying the consensus view about the chance character of all genetic mutations is to recall what biologists of the Modern Synthesis acknowledged about genetic mutations, namely their possible causes, the probability of their occurrence at different levels, and their relation to environmental conditions and the evolutionary process (in particular, see Dobzhansky 1951 [1937], ch. 2, 3; Simpson 1953, ch. 3, 4; Dobzhansky et al 1977; for further references, see note 3).

First, before the discovery of the double helix structure of DNA, biologists of the Modern Synthesis had already recognized that mutations are not always caused by errors in the normal operation of intracellular processes, but can also be induced by physical and chemical agents (e.g., ionizing radiation, temperature changes, and chemical substances like mustard gases).

Second, they also acknowledged that mutations are not equally probable across the entire genome. As Drake says: "although mutations are poorly predictable, they have already been observed to be nonrandom in one important way from the earliest decades of mutation research (the 1920s through the 1940s), mutation rates were observed to vary greatly across different eukaryotic genes" (Drake 2007, 8203). Biologists thus acknowledged that some genes are more mutable than others and that mutation rates can change in relation to the presence of physico-chemical agents and in relation to the life stages of the organism. Furthermore, they also knew that the probability of different types of genetic changes is unequal across the genome and at a specific genomic site.

After the discovery of the structure of DNA around the 1950s, biologists of the Modern Synthesis further recognized that because of the physico-chemical properties of the nucleotide sequence, some genomic sites are more mutable than others (at least ten times more, i.e., the so-called "hot-spots" of mutation; see Benzer and Freese 1958; Benzer 1959, 1961, 1962). They knew, for instance, that since cytosine is chemically unstable, the probability for a mutation to occur is higher in genomic regions rich in this nucleotide base. They also admitted that some mutagens tend to provoke mutations in some specific genomic regions more than in others, like ultraviolet radiation that tends to alter DNA sequences where thymine occurs repeatedly.

Third, not only did they admit that the probability of mutations can differ in different organisms of the same strain, in different strains of the same species, and amongst different species, but they also admitted that the mutation rate can be under genetic control through the activity of some specific genes, called "modifiers," that regulate (enhancing or decreasing) the mutation rates of other genes. Moreover, when considering genetic mutations from the evolutionary point of view, biologists of the Modern Synthesis even argued that the genetic regulation of mutation rates is the result of the evolutionary history of the group of organisms concerned and that it could be adaptively fine-tuned in relation to environmental conditions (e.g., see Dobzhansky 1951 [1937], 60-61, 74; Simpson 1953, 113). ${ }^{7}$

The idea of adaptive regulation of mutation rates spread even though it was a controversial hypothesis (e.g., see Dobzhansky 1951 [1937], 63). But biologists of the Modern Synthesis did not think that a mutation has the same probability of being beneficial, deleterious or neutral. The chances that a mutation can have a particular adaptive value depend on the genomic context in which it occurs, the environmental conditions of the organism, and the impact of the modification at the genomic level. In fact, Fisher demonstrated mathematically that a small genetic alteration has a greater chance of being beneficial than a larger one, which is more likely to be deleterious or even lethal (Fisher 1999 [1930]).

Now, since the biologists of the Modern Synthesis knew that mutations can be induced by physicochemical mutagens increasing the mutation rate, they would not have disputed the possibility that an adverse environmental condition (e.g., a sudden temperature change) could trigger an increased mutation rate across the entire genome of organisms belonging to a given population. Such a case is significant because it illustrates what the Modern Synthesis acknowledged about the occurrence of "chance mutation." First, they acknowledged a causal connection between environmental factors, which can be considered as the selective forces at play, and the increase of mutation occurrences at that moment: a global increase of mutation rate is here caused by the temperature change. Second, they accepted that mutations occur more frequently 
when they can provide some advantage to the organisms concerned, i.e., in a stressful environmental condition. With a high mutation rate, a beneficial mutation is more probable to occur quickly than in a situation where the mutation rate is lower. Thus, they also acknowledged the possible correlation, causal or not, between the probability of mutations and the population's need for variability. ${ }^{8}$ Finally, biologists of the Modern Synthesis also claimed that when organisms are poorly-adapted to the new stressful environmental condition, the probability of an advantageous mutation is greater than the probability of a deleterious one (e.g., see Simpson 1984 [1944], 55-56; Dobzhansky et al 1977, 66). ${ }^{9}$

But despite acknowledging these mutational biases, the biologist of the Modern Synthesis did not give up the claim that mutations occur by "chance" with respect to adaptation. One wonders in what sense did they consider DNA changes as "chance mutations," that is, how did they understand the notion of chance used to describe genetic mutations? Are the mutations induced by environmental factors like in the situation above still a matter of chance? In order to resolve this problem let us turn to what biologists of the Modern Synthesis acknowledged about genetic mutations, i.e., the mutational bias just listed above, and the diverse formulations they provided of the notion of "chance mutation" (see Section 2).

\section{Looking for the Modern Synthesis' Consensus View}

Let us examine the formulations that belong to the first group described earlier. It is worth beginning with Dobzhansky's way of expressing the idea of "chance mutation" because he defines it positively and explicitly refers to the physico-chemical processes involved in genetic mutations.

Dobzhansky says that "mutation is a random process with respect to the adaptive needs of the species" (Dobzhansky 1970, 65). If we were to understand his use of the term "random" in the strictly mathematical sense meaning "equally probable" and "independent" (both statistically and causally), then Dobzhansky would seem to suggest that a "random" mutation is one that has the same probability of being advantageous, deleterious or neutral. Thus, there would be no causal or statistical connection between its adaptive value (i.e., the adaptive value of the result produced by the mutational process) and the adaptive needs of the organisms concerned - or, in probabilistic terms, between the probability of a mutation occurring and its probability of being beneficial. However, as we have just seen, biologists of the Modern Synthesis did not conceive mutations to be equally beneficial, deleterious or neutral. Moreover, they claimed that the probability of developing an advantageous mutation is higher when organisms are faced with adverse environmental conditions to which they are not adapted, and when increased variability would be useful for their survival and reproduction. Therefore, the notion of chance used by biologists of the Modern Synthesis is not synonymous with the notions of "equal probability" and "independence."

We could reach the same conclusion by analyzing Dobzhansky's formulation in the light of the empirical situation described above. First, if selective environmental features (e.g., temperature change) trigger mutations, then a causal connection exists between the selective environmental factors and the probability of mutation. Second, if mutations occur more frequently in stressful conditions when they can be useful for the organisms concerned, then the probability of mutations occurring and the need for variability seem to be connected, at least statistically. Third, if advantageous mutations are more probable when the organisms are poorly adapted to the new environmental condition, then another connection, at least statistical, seems to link the adaptive value of mutations and the adaptive needs of the organisms concerned. All these elements show that if Dobzhansky's definition of chance mutations were understood in a strict mathematical sense, he would then be in contradiction with what biologists of the Modern Synthesis acknowledged about mutations.

Let us now consider the formulations belonging to the second group. Dobzhansky himself defines mutations as "random changes because they occur independently of whether they are beneficial or harmful, and therefore they are a disordering process" (Dobzhansky et al 1977, 12). I do not intend to examine this formulation in detail, but it seems evident that its analysis would lead to the same conclusion as above and would contradict what the biologists of the Modern Synthesis recognized about the mutational process. 
Turning to the third group, Simpson's formulation of the chance character of genetic mutations is particularly interesting. He clearly states that the term "random" cannot be used according to its strict mathematical meaning. He writes:

"Mutations are not random in the full and usual sense of the word or in the way some early Darwinists unrealistically considered as fully random the variation available for natural selection. [...] There is, on the one hand, a randomness as to where and when a mutation will occur": mutations can be said to be "statistically random", even if their source is not "a wholly random reaction [...] On the other hand, the term 'randomness' as applied to mutation often refers to the lack of correspondence of phenotypic effect with the stimulus and with the actual or the adaptive direction of evolution. Heat-induced mutations do not produce phenotypic change related to heat tolerance" (Simpson 1953, 86-87, my emphasis).

Simpson's approach to defining and explaining the chance character of mutations does not seem to contradict the mutational biases acknowledged by biologists of the Modern Synthesis and the empirical situation described above. Simpson clearly states that mutations are not fully random and he does not deny that there could be some connection, at least statistical, between mutation and adaptation, that is, between the probability of a mutation occurring and its adaptive value. By looking at the causal origin of genetic mutation, Simpson states that even if mutations are caused by some selective agent (e.g., a temperature change), they are not specifically provoked with a view to the adaptation of the organisms concerned to some given environmental conditions, i.e., there is no specific relation from cause to effect between the adaptive utility of a mutation and its occurrence in a given environment. In probabilistic terms, this means that there is no specific causal connection between the probability of a mutation being beneficial (in a given environment) and the probability of it occurring (in this environment).

Simpson's definition of "chance mutation" concords with the Modern Synthesis and Darwin's most influential understanding of "spontaneous," "accidental" or "chance" variations (see note 4). I therefore argue that it corresponds to the Modern Synthesis' consensus view about the chance character of genetic mutations. Let us now define it with greater precision.

\section{5. "Evolutionary Chance" Mutations}

The Modern Synthesis's consensus view on mutation can be called "evolutionary chance mutation," which may be defined using a slight reformulation of Millstein's definition of "random mutation" that is opposed to the notion of "directed mutation" (Millstein 1997). ${ }^{10}$ A genetic mutation is a matter of chance from the evolutionary perspective - or is a matter of "evolutionary chance" - if and only if it is not specifically caused in an (exclusively) adaptive way by a physico-chemical process in response to environmental conditions. ${ }^{11}$

Thus, on the one hand, a mutation is "specifically caused" if and only if it is part of a local increase of mutation rate, i.e., targeted at some particular region or site of the genome. On the other hand, a mutation is said to be caused "in an (exclusively) adaptive way" if and only if, based on the characteristics of the physico-chemical process provoking it, its probability of being beneficial is clearly higher than the probability of other deleterious or neutral mutations in the same given environment. Therefore, a mutation is "specifically caused in an (exclusively) adaptive way" - in other words, it is a "directed" mutation - if and only if it is part of a local increase of the mutation rate and the physico-chemical process causing it clearly makes the probability of a beneficial mutation higher than the probability of other deleterious or neutral mutations in the same environment. Following Millstein, this means that if a mutation is not specifically caused by a physico-chemical process in response to environmental conditions, or if it is specifically caused, but not in an (exclusively) adaptive way, it is an "evolutionary chance" mutation. Obviously, if a mutation is not caused in response to environmental conditions, it is also an "evolutionary chance" mutation. 
The notion of "evolutionary chance mutation" can be characterized in probabilistic terms by explicitly defining the necessary and sufficient conditions for a mutation to be "directed," and then defining them in the negative. A mutation is "directed" if and only if it fulfills the two following conditions:

(1) It is more probable in an environment where it is beneficial than in another environment where it is deleterious or neutral

(2) It is clearly more probable in an environment where it is beneficial than other deleterious or neutral mutations (in the same environment) ${ }^{12}$

Condition (1) pertains to the probability of a mutation in different environmental conditions and refers to the fact that the causal process that produces a "directed" mutation provokes a local increase of the mutation rate, which is targeted at the genomic region or site where the potentially adaptive mutation would have to occur. Condition (2) pertains to the probability of a beneficial mutation with respect to the probability of other deleterious or neutral mutations in the same environment and refers to the fact that the causal process that produces a "directed" mutation tends to provoke mutations that are beneficial for the organisms concerned in their environment. On this ground, I conclude that the necessary and sufficient conditions for an "evolutionary chance" mutation can be formulated as follows:

A mutation is a matter of "evolutionary chance" if and only if it is not "directed," i.e., if and only if it does not fulfill at least one of the two conditions to be a "directed" mutation

Thus far I have shown that the notion of "evolutionary chance mutation" corresponds to the Modern Synthesis' consensus view about the character of all genetic mutations. Next I will recall the main events that have marked the debate over the character of genetic mutations (Section 6) and introduce the mutator mechanisms discovered in the last thirty years (Section 7). Then, after introducing and criticizing Jablonka and Lamb's analysis of different kinds of genetic mutations and the challenge they address to the Modern Synthesis consensus view (Sections 8-9), I will provide a definitive argument for the "evolutionary chance" character of all genetic mutations (Sections 10-11).

\section{What is New about Genetic Mutations?}

What I propose to call the "evolutionary chance" character of genetic mutations (i.e., the Modern Synthesis' consensus view) was, in the 1930s, a widespread assumption shared by geneticists and evolutionary biologists, especially with regard to variations arising in higher organisms. However, there was no experimental evidence to support this idea (Sniegowski and Lenski 1995). Surprisingly, the first demonstration that provided decisive support for the idea of "evolutionary chance mutation" came from experiments on bacteria, which were then a topic of debate between the Lamarckians and the proponents of the Modern Synthesis (i.e., the "directed" and the "evolutionary chance" character of genetic mutations). In 1943, Luria and Delbrück observed that cells resistant to viral infection appeared when a population of Escherichia coli bacteria was placed on agar plates containing a virus (a lytic phage). They examined the statistical distribution of resistant mutant cells and, on this basis (i.e., by performing what they called a "fluctuation test"), argued that mutations for virus resistance arose spontaneously prior to exposure to the selective agent, i.e., during the growth phase, without any inductive role for the virus. This result was considered as evidence for the Modern Synthesis' consensus view about genetic mutations. ${ }^{13}$ In the 1940s and 1950s, other experiments confirmed Luria and Delbrück's result: Newcombe's respreading test (1949), J. Lederberg and E. Lederberg's replica plating (1952), and Cavalli-Sforza and J. Lederberg's sib selection experiment (1956).

At the end of 1980s, Cairns and his colleagues repeated Luria and Delbrück's fluctuation test and claimed to have overturned its result (Cairns et al. 1988). The possibility of a "product-oriented form of mutation" (i.e., the hypothesis of "directed mutation" or "Lamarckian bacteria") reappeared. ${ }^{14}$ Cairns and 
his colleagues defied the Modern Synthesis by suggesting that bacteria were able to "sense" their environment in order to produce beneficial mutations, i.e., to "direct" mutations in order to survive and reproduce. ${ }^{15}$ Their paper triggered a large debate during the 1990s between proponents of the "directed mutation" hypothesis and partisans of the Modern Synthesis' consensus view. Nevertheless, the former could not produce any compelling experimental demonstrations to back up their claim. Moreover, partisans of the Modern Synthesis provided several alternative explanations in terms of mechanisms for apparent cases of "directed" mutation, describing fundamentally different phenomena consistent with the idea of "evolutionary chance mutation." Despite the fact that most biologists then stopped speaking about "directed mutation," some (e.g., Shapiro 1995, 373) reformulated the hypothesis as "adaptive mutation," an ambiguous term which "sits uneasily between Lamarckian and Darwinian connotations" (Sniegowski and Lenski 1995, 566). ${ }^{16}$

Nowadays, biologists commonly use the expression "adaptive mutations" to designate beneficial mutations occurring in populations characterized by a high mutation rate in stressful environmental conditions ("mutator" populations, Taddei 1995). Even though such defined mutations fit the Modern Synthesis' consensus view, some biologists, historians and philosophers of biology still argue that their discovery is a big challenge to it (Shapiro 2005; Wright et al. 1999, Wright 2000; Sternberg 2002; Keller 2000; Jablonka and Lamb 2005). Some have even gone so far as to claim that the discovery of the genetic regulation of mutation rates by some molecular mechanisms like mutators (see note 2) in relation to stressful environmental conditions constitutes a biological revolution - even a "quantum" one (Shapiro 1999).

Jablonka and Lamb are paradigmatic exponents of the challenge to the Modern Synthesis' consensus view. They claim that Darwinian evolution must include instructive Lamarckian processes in order to account for mutations due to certain kinds of mutator mechanisms. It is worth noting, however, that their aim is not to challenge the whole Modern Synthesis; they simply question the classic tenet about the chance character of all genetic mutations with respect to adaptation, and claim that a better and Extended Synthesis is possible by incorporating some Lamarckian ideas about the origin of hereditary variation. Since Jablonka and Lamb's empirical and conceptual analysis of genetic mutations is the most recent challenge to the Modern Synthesis's notion of "evolutionary chance," their claim will now be the target of my criticisms.

\section{Mutator Mechanisms}

Let us now turn to the recently discovered mutator mechanisms themselves. This will allow us to better understand why their discovery is thought of as a challenge to the Modern Synthesis' consensus view about mutations.

Recent experimental research, especially in microbiology, ${ }^{17}$ has shown that certain molecular mechanisms can regulate mutation rates in response to changes in an organism's environment. Biologists use the expression "mutator mechanisms" to designate the complex enzymatic machineries that can react to selective forces by producing increased mutation rates. Biologists distinguish different types of mutator mechanisms, which, depending on the following features (Giraud et al. 2001), can be either:

"Constitutive," i.e., once it has appeared following an environmental stress and become fixed in a population, it stays turned on in individual organisms and their offspring, even in the absence of any environmental stimulus; or,

"Induced," i.e., temporarily activated in response to more or less specific stimuli of stress.

Moreover, in addition to being constitutive or induced, a mutator mechanism can also be:

"Global," i.e., it provokes a global increase of mutation rate, which is not targeted at any particular region or site of the genome; or, 
"Local," i.e., it provokes a local increase of mutation rate, targeted at some specific genomic region or site.

These categories, along with certain experimental results, allow biologists to distinguish four types of mutator mechanisms:

a) Constitutive, provoking a global increase of mutation rates, e.g., the inactivation of one or more steps of the repair system of DNA replication ${ }^{18}$

b) Constitutive, causing a local increase of the mutation rate, e.g., "hot-spots" of hypermutability targeted at some specific region or site of the genome ${ }^{19}$

c) Induced, provoking a global increase of the mutation rate, e.g., the so-called "SOS system," which is temporarily induced by stressful conditions ${ }^{20}$

d) Induced, causing a local increase of mutation rate, e.g., the local hypermutability caused by starvation

Since the fourth type of mutator mechanisms is the most controversial, and since Jablonka and Lamb claim that the mutations it provokes are not "random" (Jablonka and Lamb 2005, 97), let us examine it in detail through the following example: the induced and local hypermutability observed in E. coli bacteria LeuBwhen in leucine starvation (Wright et al. 1999, Wright 2000; see also Jablonka and Lamb 2005).

In order to better understand how this particular mutator mechanism works, it is important to recall the well-known physiological mechanism in bacteria that regulates the expression of genes involved in the production of amino acids in response to environmental conditions. This mechanism allows for the conservation of the precious reserves of nutrients as well as the activation and derepression of systems essential to the bacterium's survival (Bruhat et al. 1999). For instance, starvation for lack of leucine specifically targets the derepression of the leucine (leu) operon involved in the production of this amino acid.

In their study, Wright and her colleagues used E. coli bacteria $\mathrm{K} 12 \mathrm{LeuB}^{-}$, a strain that is unable to produce the amino acid leucine because of a mutation in the leuB gene on the leu operon. They placed this strain of bacteria in leucine starving conditions: as expected, they observed a series of specific metabolic activities for the activation of leu operon and the increase of its transcription rate. They also observed an increase in the mutation rate targeted at the leu operon including the gene leuB. In other words, Wright and her colleagues noticed that when bacteria that are unable to synthesize leucine are in leucine starvation the mutation rate at the level of the leu operon, involved in the production of leucine, is higher than in normal environmental conditions (i.e., in a milieu rich in leucine). This specific molecular phenomenon increases the probability of mutations affecting the leu operon. More importantly, however, it also increases the probability of the beneficial mutation from LeuB- to $\mathrm{LeuB}^{+}$, which would give the bacteria the ability to produce leucine and, thereby, survive and reproduce in adverse environmental conditions.

On the basis of these results, Wright and her colleagues suggested that the induced and local hypermutability was an adaptive result of evolution by natural selection, that is, an adaptive response to adverse environmental conditions or, to be more precise, to amino acid starvation. However, if we pay attention to the details of the physiological response they observed, their adaptive hypothesis is far from obvious. In fact, it seems more plausible that the increased mutation rate at the level of the leu operon is a simple by-product of its derepression and the activation of gene expression at this site. The response to leucine starvation observed in E. coli LeuB- bacteria can be described as follows (see Figure 1). The environmental stress (leucine starvation) triggers the derepression of the leu operon and activates its expression. The structure of the DNA molecule at the level of the leu operon then changes: the derepression provokes the formation of the transcription bubble, exposes single non-transcribed DNA strands, drives negative super-coils, and triggers the formation of some secondary structures characterized by base mispairing. All these changes at the level of the leu operon make this site vulnerable to mutational events. And thus, the mutation rate locally increases when bacteria are in leucine starvation (Merlin 2009). 


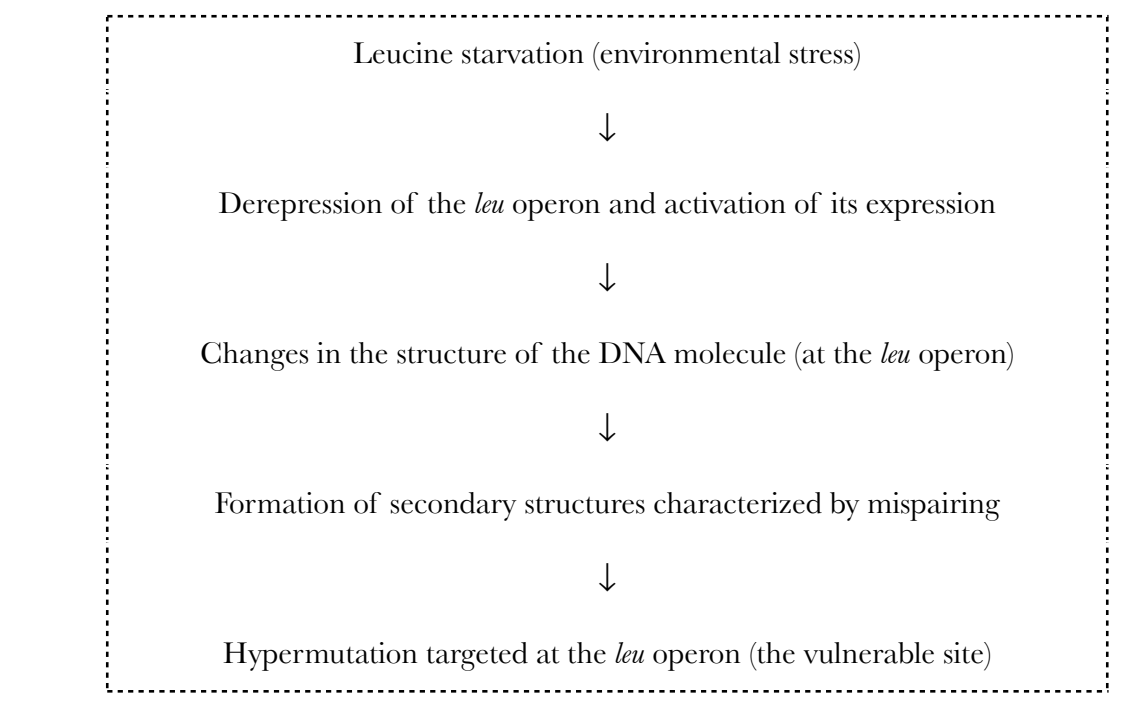

Figure 1 - The main steps of the response to leucine starvation observed in $E$. coli LeuB- bacteria by Wright and her colleagues.

Jablonka and Lamb (2005) explicitly deny the empirical applicability of the Modern Synthesis' consensus view in the case of mutations due to this type of mutator mechanisms. They claim that these mutations occur in response to a stress stimulus - i.e., in response to the selective forces at play - and are targeted at the genomic site where mutations would be beneficial to the organism given its adverse environmental conditions. In other words, according to Jablonka and Lamb, mutations due to induced and local mutator mechanisms occur when and where they are needed by organisms in order to survive and reproduce in their environment. These mutations are therefore "non-random," "likely to be adaptive," "both acquired and required," that is, they are provoked by physico-chemical processes that are "both instructive and selective." For these reasons, Jablonka and Lamb conclude that we should include Lamarckian (instructive) processes into Darwinian (selective) evolution in order to explain them (Jablonka and Lamb 2005, 97, 101-102).

Having introduced and discussed the most controversial type of mutator mechanisms, which Jablonka and Lamb consider a big challenge for the Modern Synthesis' consensus view, let us now analyze Jablonka and Lamb's claims about mutations due to mutator mechanisms. This will then allow me to defend the idea that all genetic mutations are "evolutionary chance" mutations (i.e., the Modern Synthesis' paradigm).

\section{The Modern Synthesis' and Lamarck's Ideas as Seen by Jablonka and Lamb}

In their most recent book, Jablonka and Lamb (2005) examine the evolutionary contribution of what they call the "four dimensions" of evolution: genetic, epigenetic, behavioral and symbolic inheritance. They also provide a clear and exhaustive description of relatively recent discoveries about mutations and try to analyze the possible differences among mutations provoked by a variety of physico-chemical mechanisms. I will now analyze the first part of their book, focused on hereditary genetic variation, and, using empirical evidence about mutations due to mutator mechanisms, I will argue against their conceptual and empirical distinction between "random" and "non-random" mutations, as well as their finer one between "totally blind," "semi-directed" and "totally directed" mutations. I will show that Jablonka and Lamb's definitions and arguments are overly influenced by their general intention of including Lamarckian processes in Darwinian evolution. 
But before analyzing Jablonka and Lamb's account of the different kinds of genetic mutations, let us look at their description of the Modern Synthesis' consensus view and the Lamarckian conception of hereditary variation. In their most recent book, they define the classic conception of the origin of genetic hereditary variation as "totally random" or "blind" mutation and oppose it to the Lamarckian idea of "soft inheritance" or the "inheritance of acquired characters." Thus, their first claim is that the biologists of the Modern Synthesis viewed mutations as errors in DNA replication, which have nothing to do with the individual history of the organisms concerned. The idea of mutations produced in response to the adaptive needs of organisms would be foreign to the Modern Synthesis (see Jablonka and Lamb 2005, 7, 29, 33-34, 99, 87). Jablonka and Lamb then claim to oppose the Modern Synthesis' consensus view by introducing the idea of "the inheritance of genomic changes induced by environmental factors" which corresponds, according to them, to the Lamarckian notion of "soft inheritance" (see Jablonka and Lamb 2005: 7). These two claims call for some remarks.

Although Jablonka and Lamb's way of presenting the Modern Synthesis' consensus view is not wrong, it is incomplete and insufficiently analytic. Their presentation does not acknowledge the richness of what biologists of the Modern Synthesis knew about genetic mutations. Rather, Jablonka and Lamb offer what I would call a "naïve" interpretation of the Modern Synthesis' paradigm (Merlin 2009), which is based on the simplified and idealized representation of the mutation process in genetic population models and does not take into consideration the mutational biases biologists of the Modern Synthesis readily admitted (see above, Section 3).

Jablonka and Lamb also provide an ambiguous definition of the "inheritance of acquired characters," which is not antithetical with the Modern Synthesis' fundamental ideas. Their understanding of "inheritance of acquired characters" differs not only with the one August Weismann so strongly criticized (Weismann 1892 [1883], 1893 [1892]) but also with Lamarck's own conception of the origin and the character of hereditary variation.

As Haig (2006) has already stressed, Jablonka and Lamb's definition of the "inheritance of acquired characters" could equally apply to genetic mutations caused by mutagenic agents (e.g., UV radiations, chemical gases) or to some non-controversial kind of mutator mechanisms, like the activation of the SOS system in stressful environmental conditions (see above, Section 7). More importantly, Jablonka and Lamb also claim that Lamarckian processes are needed in order to account for highly biased hereditary modifications induced by the environment, no matter their adaptive value, because the Darwinian Modern Synthesis is supposedly unable to explain such modifications. However, what Jablonka and Lamb describe, controversially, as the "inheritance of acquired characters" is not at all in contradiction with the Modern Synthesis' view, which actually accounts for the origin and the character of these genetic mutations.

Moreover, Jablonka and Lamb's use of the notion of "inheritance of acquired characters" (or "soft inheritance") is historically inaccurate. The notion that Weismann attacked in the $19^{\text {th }}$ century referred to the hereditary transmission of adaptive somatic changes acquired during an organism's lifetime, which were not accompanied by any modification at the level of the hereditary genetic material. Weismann never denied that the external environmental conditions could induce modifications of the hereditary material (the germ-plasma) and believed that "the ultimate source of variation is always the effect of external influences" (Weismann, 1893 [1892], 463, emphasis in the text). ${ }^{21}$ Furthermore, according to Lamarck's conception of the origin of hereditary variation and adaptation, individual organisms change in an adaptive manner under environmental influence, that is, in order to cope with their milieu, and consequently, individual adaptation is the source of phylogenetic adaptation (i.e., at the species level). Thus, it is easy to see that Jablonka and Lamb's definition of the "inheritance of acquired characters" as "the inheritance of genomic changes induced by environmental factors" is neither a modern reformulation of what Weismann attacked nor of Lamarck's conception of hereditary variation and adaptation.

I have just underlined the limitation of Jablonka and Lamb's description of the Modern Synthesis' consensus view about genetic mutations and shown that their neo-Lamarckism is an inaccurate redefinition of Lamarck's ideas. In the next section, I will introduce their conceptual and empirical analysis of different kinds of genetic mutations, which will allow me to argue for the "evolutionary chance" character of all genetic mutations (i.e., the Modern Synthesis' consensus view). 


\section{Mutator Mechanisms: Jablonka and Lamb's Analysis Put to the Test}

Let us analyze Jablonka and Lamb's terminological and conceptual distinctions about the character of genetic mutations. The aim is to understand the distinction they make between "random" and "non random" (or "directed") mutations as well as between "totally blind," "semi-directed" and "totally directed" mutations. In their 1995 book, they claim that mutations are "directed" if and only if a "particular environmental challenge produces specific and repeatable changes in the hereditary material. The changes can be advantageous, detrimental, or neutral" (Jablonka and Lamb 1995, 57). So, all genetic mutations occurring in response to stressful environmental conditions which are not part of a general increase of mutation rate would be "directed" mutations, even if they are harmful or neutral (e.g., mutations due to the most controversial type of mutator mechanism, the induced and local hypermutability observed in $E$. coli bacteria LeuB- in leucine starvation).

In their most recent book (2005), Jablonka and Lamb propose a finer distinction between "totally directed" and "semi-directed" mutations:

"Totally directed" mutations are "reproducible adaptive changes that occur at specific sites in response to specific stimuli," such as DNA changes occurring during gene expression and cellular differentiation (89; cf., 68-70).

"Semi-directed" mutations are genetic variations "produced in stressful conditions" in "response to environmental signals," but do "not lead to a unique and necessarily adaptive response" (88; cf., 89).

Jablonka and Lamb conceive what they call "semi-directed" mutations as "somewhere between totally blind variations, which are specific neither in their nature nor in the time and site in the genome where they occur, and totally directed variations." As we have seen above, when Jablonka and Lamb talk about "totally blind" mutations, they refer to genetic mutations according to the Modern Synthesis (see Figure 2).

Thus, according to Jablonka and Lamb, the difference between what they call "random ("totally blind") and "non random" ("directed," i.e., "totally directed" and "semi-directed") lies in the specificity of their occurrence (i.e., whether or not they are part of a general increase of mutation rate) and the causal role of the environment. A mutation is "non random" if it is specific, i.e., it is part of a local increase of mutation rate, or if the environmental conditions play a causal role in its production. Therefore, the adaptive character of the mutation (the fact that it is beneficial, deleterious or neutral) does not determine whether it is "random" or "non random" ("directed"). Rather, it plays a role in distinguishing between "semi-directed" and "totally-directed" mutations, the latter being both specific and selectively advantageous.

\begin{tabular}{|l|c|}
\hline "Random" mutations & $\begin{array}{c}\text { "Totally blind" mutations (according to the Modern Synthesis) } \\
\text { "Non random" mutations }\end{array}$ \\
$\begin{array}{c}\text { "Semi-directed" mutations } \\
\text { (due to different kinds of mutator mechanisms) } \\
\text { "Totally directed" mutations }\end{array}$ \\
\hline
\end{tabular}

Figure 2 - The distinction between "random" mutations and "non random" mutations according to Jablonka and Lamb (2005). 
I agree with the choice of criteria (specificity and causality) used to distinguish between "random" and "non random" ("directed") mutations. But in the name of the Modern Synthesis' consensus view, I suggest using a third well-known criterion, namely the adaptedness of mutations, to distinguish what I call "evolutionary chance mutations" from "directed mutations" (see Millstein 1997). All mutations are "evolutionary chance" mutations since they are not genetic changes specifically produced in an (exclusively) advantageous manner in response to a given environmental challenge. Therefore, Jablonka and Lamb's definition of "directed" ("totally directed" and "semi-directed") mutations is inappropriate. I propose instead that we stick with the Modern Synthesis' consensus view and define "directed" mutations as "non evolutionary chance" mutations, that is, mutations specifically caused in an (exclusively) adaptive way by a physico-chemical process in response to environmental conditions (see above, Section 5).

Having critiqued Jablonka and Lamb's distinction between "random" and "non random" ("directed") mutations, and in order to show that the Modern Synthesis' consensus view withstand the challenge because it applies to all genetic mutations, I will now investigate the empirical applicability of the notion of "evolutionary chance" in the case of induced and local hypermutability (Wright et al. 1999), and then show the empirical and conceptual relevance of my analysis with respect to Jablonka and Lamb’s.

\section{The "Evolutionary Ghance" Character of All Genetic Mutations}

Let us return to the induced and local hypermutability studied by Wright and her colleagues in E. coli K12 bacteria and test the "evolutionary chance" character of the observed mutations. Recall that this case is one of the most controversial kinds of mutator mechanisms and according to Jablonka and Lamb it challenges the Modern Synthesis' consensus view about the chance character of genetic mutations. The hypermutability targeted at the leu operon has been observed when bacteria, which are unable to produce leucine because of a mutation of the leuB gene, are placed in a state of leucine starvation. Wright and her colleagues observed that the rate of the reverse advantageous mutation (from LeuB- to LeuB ${ }^{+}$), which allows these bacteria to produce leucine and to survive and reproduce, is higher than in normal environmental conditions (i.e., than in a milieu rich in leucine).

To show that the notion of "evolutionary chance" can be applied to mutations due to this kind of mutator mechanism, there are three well-known distinctions that are helpful in understanding the nature of these mutational phenomena. First, there is a difference between the molecular mechanism producing an increased (global or local) mutation rate and each particular mutation it produces. This is the distinction between the mutational process, which can be defined as a series of physico-chemical causally related states, occurring over time and bringing about a final physico-chemical state, and a particular mutation, which is one of the possible final results of the increased mutation rate provoked by the mutational process.

Second, different mutator mechanisms have different origins. On the one hand, a mutator mechanism (and, more generally, a mutational bias) can be the result of the evolutionary process. Thus, a mutator mechanism can be either an adaptation (i.e., an adaptive outcome of evolution by natural selection for the function it provides), a by-product of the selection for another trait, or an outcome of evolution by random genetic drift. But, on the other hand, an increased mutation rate can also be the consequence of some other physiological mechanism or the manifestation of a pathological state in organisms experiencing adverse environmental conditions (i.e., not an evolutionary result). Within these two categories of mechanisms (or biases), we can further distinguish constitutive and temporary induced mechanisms (or biases) producing a global or local increase of mutation rate. Using this categorization, we can easily identify and classify each particular physico-chemical process that produces genetic mutations (see Table 1).

Third, it is also important to keep in mind the distinction between Darwinian (Modern Synthesis') and Lamarckian conceptions of adaptation and evolution, i.e., what Dawkins calls "the selection theory" and "the instruction theory" of the origin of "adaptedness" (Dawkins 1999 [1982]).

Now, in order to explain the reasons for my defense of the Modern Synthesis' consensus view, let us apply these three fundamental distinctions to the induced and local hypermutability observed in E.coli bacteria LeuB- (Wright et al 1999). My aim is to show that mutations due to this controversial mutator mechanism are not "directed" but "evolutionary chance" mutations. I will do so by answering the following 


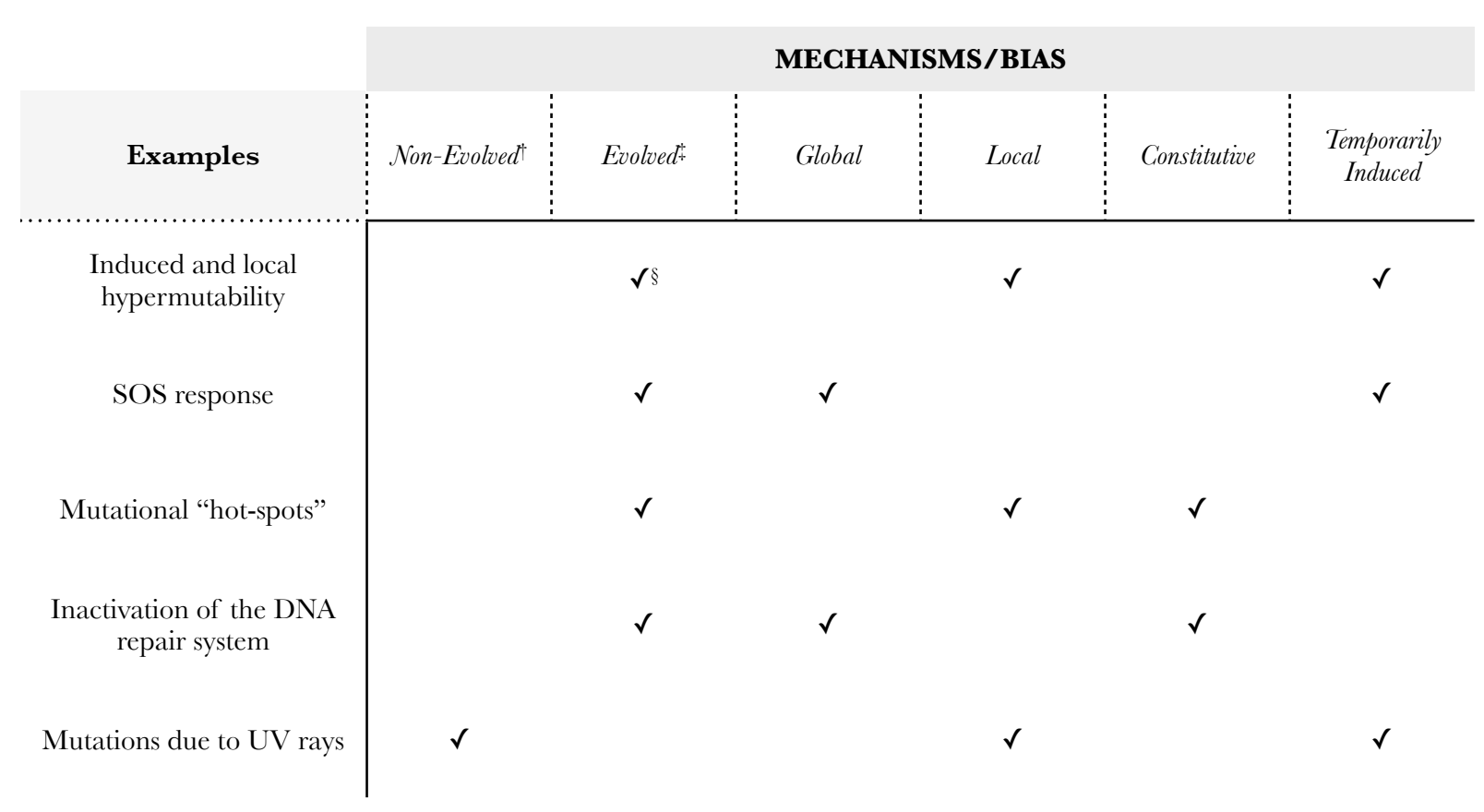

Table 1 - Mutator mechanisms and mutational bias. $\dagger$ "Non-Evolved" mechanisms and biases (i.e., due to a physiological mechanism or to a pathological state); $¥$ "Evolved" mechanisms and biases (i.e., adaptations, by-products of evolution by natural selection for another trait, or outcomes of evolution by random drift); § according to Wright et al. 1999.

question: is the reverse mutation from $\mathrm{LeuB}^{-}$to $\mathrm{LeuB}^{+}$, which allows bacteria to produce leucine when needed, specifically caused in a (exclusively) adaptive way by a physico-chemical process in response to leucine starvation or not?

The mutation from LeuB- to $\mathrm{LeuB}^{+}$is one possible result of the increased mutation rate targeted at the leu operon due to the physico-chemical state of this site along the DNA sequence. Recall that the targeted increase of mutation rate at the level of this genomic site - which is, indeed, very vulnerable to mutations is due to the presence of a transcription bubble, single non-transcribed DNA strands, negative super-coils, and secondary structures characterized by nucleotide mispairs. This physico-chemical state of the leu operon is due to its derepression and the activation of its transcription in response to leucine starvation. Thus, on the one hand, the favorable mutation allowing bacteria to produce leucine is caused by a physico-chemical process in response to a stressful environmental stimulus, and, on the other hand, it is also specifically produced as part of a local increase in the mutation rate targeted at the leu operon.

The beneficial mutation at the level of the leuB gene fulfills the first but not the second condition to be a "directed" mutation and is therefore an "evolutionary chance" mutation according to the Modern Synthesis' consensus view (see above, Section 5). The mutation from LeuB to LeuB ${ }^{+}$is more probable in an environment where bacteria are in leucine starvation than in an environment where the mutation is neutral or deleterious (e.g., in a milieu rich in leucine). This is due to the increase of the mutation rate targeted at the leu operon in response to leucine deprivation. However, the probability of this beneficial reverse mutation occurring is not higher than for other neutral or deleterious mutations in the same leucine deprived environment. In fact, Wright and her colleagues observed that this increased mutation rate is not only targeted at the leuB gene, where a mutation could allow bacteria to survive and reproduce, but at all the genes of the leu operon as well. Therefore, the reverse beneficial mutations at the level of the leuB gene may seem to be "directed" simply because it is easier to detect than mutations occurring in these other genes, which may either grow slower or be negatively selected. Thus, since bacteria carrying the reverse beneficial 
mutation from LeuB- to $\mathrm{LeuB}^{+}$are positively selected and contribute to the next generation, they can be easier to detect and quantify than bacteria with other mutations.

My claim is not that "directed" mutations with respect to adaptation are physically impossible; I simply claim that mutations due to different types of mutator mechanisms, even the most controversial ones, are "evolutionary chance" mutations, and that "directed" mutations - i.e., specifically caused in an (exclusively) adaptive way by a physico-chemical process in response to changes in environmental conditions - have yet to be observed. I also argue that the existence of "directed mutation" mechanisms, as I define them, is quite implausible. In fact, the adaptive character of a genetic mutation depends on a complex set of environmental factors (e.g., the molecular context in which the mutation occurs, the organism's internal and external environment, the other organisms present in the same environment, etc.), which are neither stable nor spatially and temporally homogeneous. For all these reasons, the evolution of a mutator mechanism producing "directed" mutations with respect to adaptation is very implausible.

\section{The Conceptual and Empirical Value of the Notion of "Evolutionary Chance"}

So, why is my distinction between "evolutionary chance mutation" and "directed mutation" of greater conceptual and empirical relevance than Jablonka and Lamb's distinction between "random" and "non random" mutations (see Figure 2)? There are three reasons for this, related to the three distinctions introduced earlier, which, I believe, contribute decisively to the resolution of the old debate over the character of genetic mutations.

First, the Modern Synthesis' definition of "evolutionary chance mutation" highlights the difference between the mutator mechanism and the mutations it produces. On the one hand, the particular mutations produced by the activation of a mutator mechanism in response to stressful environmental conditions pertain to the individual history of the organisms concerned. These mutations are the result of a physiological mechanism operating inside the cell and can be advantageous, disadvantageous, or neutral depending on the individual organism and its environment. But on the other hand, the mutator mechanisms themselves, which provoke an increase of mutation rate in response to environmental conditions, can be shown to be an adaptive result of the population's evolutionary history (as is the case for the inactivation of the repair system, the "hot-spots" hypermutability, and the SOS response). This means that the mutator mechanism has some adaptive value for individuals or populations. In a case where a mutator mechanism happens to provoke some favorable mutation, it may be positively selected at the individual level since it may provide an advantage to some lucky individual organism and its offspring (because there is selection for the favorable mutation). But a mutator mechanism could also provide an advantage to populations since it may increase the probability that a population adapts more rapidly than others to their adverse environmental conditions. ${ }^{22}$ In both scenarios (i.e., individual selection and group selection), the mutator mechanism is an evolutionary adaptive outcome producing "evolutionary chance" mutations.

The second reason in favor of the notion of "evolutionary chance mutation" stems from the distinction between different types of mutator mechanisms and biases. As I have already noted, a mutator mechanism can be constitutive or (temporarily) induced, it can provoke a global or local increase in the mutation rate, it can be an evolutionary (adaptive) result, a consequence of some other physiological mechanism, or simply the manifestation of a pathological condition in a stressful environment. These distinctions allow us to organize all "evolutionary chance" mutations in a continuous and progressive manner ranging from a strictly mathematical conception of "randomness" to the notion of evolutionary "directedness." My claim is that all "evolutionary chance" mutations can be ordered in this way because they all have some kind of bias. Their differences lie in the character, the degree and the origin of their respective mutational mechanisms and biases. In fact, no genetic mutation is strictly "random" (i.e., equally probable and independent from one another and from environmental circumstances) or "directed" to a beneficial result.

Third, the Modern Synthesis' consensus view about the character of genetic mutations emphasizes the fundamental difference between "the selection theory" and "the instruction theory" of the origin of adaptedness (Dawkins 1999 [1982], 173). According to "the selection theory," organisms' adaptedness to their environment originates in an initial pool of variation and improves over time by natural selection. 
According to "the instruction theory," however, the environment directly produces the good variation, and adaptedness is improved by a specific and adaptive causal connection between organisms and the environment. Since, insofar as genetic variations are concerned, there is no empirical evidence to support the possibility of instructively acquired characters, I agree with the Modern Synthesis' view and conclude that selection, rather than instruction, explains the possible adaptedness of both mutator mechanisms and their mutations.

Jablonka and Lamb claim that mutator mechanisms - in particular mechanisms producing mutations in response to environmental stimuli and increasing the mutation rate locally - are both selective (Darwinian) and instructive (Lamarckian) since they are the result of the evolutionary history of the population concerned and produce mutations when and where organisms need them. They then conclude from this that Lamarckian processes must be included in the Darwinian conception of evolution. In this paper, I have shown that Jablonka and Lamb's claims cannot apply if the terms "instructive" and "Lamarckian" refer to a modern and appropriate reformulation of Lamarck's view about the origin of hereditary variation and adaptation.

\section{Conclusion}

I have shown that all genetic mutations - including mutations specifically occurring in response to changes in environmental conditions (i.e., due to induced and local mutator mechanisms) - can be considered as chance mutations according to the notion I call "evolutionary chance." This notion corresponds to the Modern Synthesis' consensus view about the origin and the character of all genetic mutations. Contrary to Jablonka and Lamb (2005), and in keeping with Millstein (1997), I have argued that the discovery of the genetic regulation of mutation rates in relation to the selective forces at play does not represent any challenge to the Modern Synthesis' idea of "evolutionary chance mutation." In so doing, I criticized Jablonka and Lamb's definitions and distinctions, in particular their conception of a Lamarckian process and of the Modern Synthesis' consensus view about the origin of genetic mutations. I also provided an argument in favor of some alternative definitions and distinctions, which I believe are conceptually and empirically more appropriate than Jablonka and Lamb's. Actually, if correctly appreciated, my argument could have a positive impact on research in evolutionary biology since it provides practicing biologists with further reasons to think that recent findings on genetic mutations are not so revolutionary as they seem and, above all, that these findings are not in conflict with the Modern Synthesis consensus view. Thus construed positively, my argument liberates evolutionary biologists from a purported controversy and thereby widens the scope of their research in the field.

To conclude, I think the Modern Synthesis theory of evolution needs to recognize the importance of some recent advances in the study of the origin of genetic mutations. Recent experiments on microorganisms are of particular importance since they show that mutations not only occur during the exponential growing phase as is already known, but also during the stationary phase due to the presence of environmental stresses. Thus, contrary to one of the principal assumptions held by the Modern Synthesis, genetic variability does not always precede selective pressures, but can also occur in response to them. To me, this novelty can be exhaustively explained by a more inclusive version of the Modern Synthesis - an Extended Synthesis (Pigliucci and Müller 2010) -, which would thereby preserve its fundamental notions, in particular "evolutionary chance mutation," as well as its empirical validity and capacity to explain all biological phenomena observed until now.

\section{Literature cited}

Beatty, J. 1984. Chance and natural selection. Philosophy of Science 51: 183-211.

Beatty, J. 1987. Introduction. In The Probabilistic Revolution: Volume II. Ed. by L. Krüger, G. Gigerenzer and M.S. Morgan. Cambridge: MIT Press.

Beatty, J. 2006. Chance variation: Darwin on orchids. Philosophy of Science 73:692-641. 
Beatty, J. 2008. Chance variation and evolutionary contingency: Darwin, Simpson (The Simpsons) and Gould. In Oxford Handbook of the Philosophy of Biology. Ed. by M. Ruse. Oxford: Oxford University Press.

Benzer, S. 1959. On the topology of genetic fine structure. Proceedings of the National Academy of Sciences USA 45: 1607-1520.

Benzer, S. 1961. On the topology of genetic fine structure. Proceedings of the National Academy of Sciences USA 47: 403-415.

Benzer, S. 1962. The fine structure of the gene. Scientific American 206: 70-84.

Benzer, S. and E. Freese. 1958. Induction of specific mutations with 5-bromouracil. Proceedings of the National Academy of Sciences USA 44: 112-119.

Bruhat, A., Jousse, C., and P. Fafournoux. 1999. Amino acid limitation regulates gene expression. Proceedings of Nutrition Society 58: 625-632.

Cairns, J and P.L. Foster. 1991. Adaptive reversion of a frameshift mutation in Escherichia coli. Genetics 128: 695-701.

Cairns, J., Overbaugh, J. and S. Miller. 1988. The origins of mutants. Nature 355: 142-145.

Caporale, L.H. (ed) 1999. Molecular Strategies in Biological Evolution. New York: Annals of the New York Academy of Science (870).

Caporale, L.H. 2000. Mutation is modulated: implications for evolution. BioEssays 22: 388-395.

Caporale, L.H. 2003. Natural selection and the emergence of a mutation phenotype: an update of the Evolutionary Synthesis considering mechanisms that affect genome variation. Annual Review of Microbiology 57: 467-485.

Cavalli-Sforza, L.L. and J. Lederberg. 1956. Isolation of pre-adaptive mutants of bacteria by sib-selection. Genetics 41: 367-381.

Darden, L. 2006. Reasoning in Biological Discoveries: Essays on Mechanisms, Interfield Relations, and Anomaly. Cambridge: Cambridge University Press.

Darwin, G. 1859. On The Origin of Species By Means of Natural Selection or The Preservation of Favoured Races in The Struggle For Life. London: John Murray (first edition).

Darwin, G. 1868. The Variation of Animals and Plants Under Domestication. 2 vols. London: John Murray (first edition).

Darwin, F and A.C. Seward. (eds) 1903. More Letters of Charles Darwin. Volume I. London: John Murray.

Dawkins, R. 1999 [1982]. The Extended Phenotype. The Long Reach of the Gene. Oxford: Oxford University Press.

Dobzhansky, T. 1951 [1937]. Genetics and The Origin of Species. New York: Columbia University Press.

Dobzhansky, T. 1970. Genetics of the Evolutionary Process. New York: Columbia University Press.

Dobzhansky, T., Ayala, F.J., Stebbins, G.L. and J.W. Valentine. 1977. Evolution. San Francisco: WH Freeman \& Company.

Drake, J.W. 2007. Mutations in clusters and showers. Proceedings of the National Academy of Sciences USA 104: 8203-8204.

Eble, G. 1999. On the dual nature of chance in evolutionary biology and paleobiology. Paleobiology 25: 75-87.

Fisher, R.A. 1999 [1930]. The Genetical Theory of Natural Selection: a Complete Variorium Edition. Oxford: Oxford University Press.

Foster, P.L. 2000. Adaptive mutation: implications for evolution. BioEssays 22: 1067-1074.

Foster, P.L. 2004. Adaptive mutation in Escherichia coli. Journal of Bacteriology 186: 4846-4852.

Galhardo, R.S., Hastings, P.J. and Rosenberg S.M. 2007. Mutation as a stress response and the regulation of evolvability. Critical Reviews in Biochemistry and Molecular Biology 42: 399-435.

Giraud, A., Radman, M., Matic, T. and F. Taddei. 2001. The rise and the fall of mutator bacteria. Current Opinion in Microbiology. 4: 582-585.

Haig, D. 2006. Weismann rules! Epigenetics and Lamarckian temptation. Biology and Philosophy 22: 415-428.

Haldane, J.B.S. 1966 [1932]. The Causes of Evolution. New York: Cornell University Press. 
Hall, B.G. 1998. Adaptive evolution that requires multiple spontaneous mutations. I. Mutations involving an insertion sequence. Genetics 120: 887-897.

Hastings, P.J., Slack, A., Petrosino, J.F. and S.M. Rosenberg. 2004. Adaptive amplification and point mutation are independent mechanisms: Evidence for various stress-inducible mutation mechanisms. PLoS Biology 2(12): e399.

Huxley, J. 1948 [1942]. Evolution. The Modern Synthesis. London: George Allen \& Unwin Ltd.

Huxley, J. 1953. Evolution in Action. New York: Harper \& Brothers.

Jablonka, E. and M. Lamb. 1995. Epigenetic Inheritance and Evolution. Oxford: Oxford University Press.

Jablonka, E. and M. Lamb. 2005. Evolution in Four Dimensions. Genetic, Epigenetic, Behavioral, and Symbolic Variation in the History of Life. Cambridge: MIT Press.

Keller, E.F. 2000. The Century of the Gene. Cambridge: Harvard University Press.

Lederberg, J. and E. Lederberg. 1952. Replica plating and indirect selection of bacterial mutants. Journal of Bacteriology 63: 399-406.

Leigh, E.G. Jr. 1973. The evolution of mutation rates. Genetics (Suppl) 73: 1-18.

Lennox, J.G. 2010. Darwinism. In The Stanford Encyclopedia of Philosophy (Fall 2010 Edition), Ed. by E.N. Zalta: http://plato.stanford.edu/entries/darwinism/.

Lenski, R.E. and J.E. Mittler. 1993. The directed mutation controversy and neo-Darwinism. Science 259: 188-194.

Luria, S.E. and M. Delbrück. 1943. Mutations of bacteria from virus sensitivity to virus resistance. Genetics 28: 491-511.

Mayr, E. 1942. Systematics and the Origin of Species. New York: Columbia University Press.

Mayr, E. 1977 [1963]. Populations, Species, and Evolution. Cambridge, Massachusetts: The Belknap Press of Harvard University Press.

Merlin, F. 2009. Le hasard et les sources de la variation biologique: analyse critique d'une notion multiple. Doctoral dissertation. University of Paris 1 Panthéon-Sorbonne.

Miller, J.H. 1996. Spontaneous mutators in bacteria: insights into pathways of mutagenesis and repair. Annual Review of Microbiology 50: 625-643.

Millstein, R.L. 1997. The Chances of Evolution: An Analysis of the Roles of Chance in Microevolution and Macroevolution. Doctoral dissertation. Department of Philosophy, University of Minnesota.

Moxon, E.R., Rainey, P.B., Nowak, M.A. and R.E. Lenski. 1994. Adaptive evolution of highly mutable loci in pathogenic bacteria. Current Biology 4: 24-33.

Moxon, E.R., Bayliss, G. and D. Hood. 2006. Bacterial contingency loci: the role of simple sequence DNA repeats in bacterial adaptation. Annual Review of Genetics 40: 307-333.

Newcombe, H. 1949. Origin of bacterial variants. Nature 164: 150-151.

Pigliucci, M. and G.B. Müller. (eds) 2010. Evolution - the Extended Synthesis. Cambridge, MA: MIT Press.

Radman M. 1973. Phenomenology of an inducible mutagenic DNA repair pathway in Escherichia coli: SOS repair hypothesis. In: Molecular and Environmental Aspects of Mutagenesis. Ed. by L. Prakash, F. Sherman, M. Miller, G. Lawrence and H.W. Tabor. Springfield: Charles C. Thomas.

Radman, M., Taddei, F. and I. Matic. 2000. Evolution-driving genes. Research in Microbiology 151: 91-95.

Radman, M. and R. Wagner. 1986. Mismatch repair in Escherichia coli. Annual Review of Genetics 20: 523-538.

Rensch, B. 1959. Evolution Above the Species Level, London: Methuen and Co Ltd.

Rosenberg, S.M. 2001. Evolving responsively: adaptive mutation. Nature Reviews Genetics. 2: 504-515.

Sarkar, S. 1991. Lamarck contre Darwin, reduction versus statistics : Conceptual issues in the controversy over directed mutagenesis in bacteria, In Organism and the Origins of Self. Ed. by A.I. Tauber. Netherlands: Kluwer Academic Publishers.

Shapiro, J.A. 1984. Observations on the formation of clones containing araB-lacZ cistron fusion. Molecular Genetics and Genomics. 194: 79-90.

Shapiro, J.A. 1995. Adaptive mutation: who's really in the garden? Science 268: 373-374.

Shapiro, J.A. 1999. Genome system architecture and natural genetic engineering in evolution. In Molecular Strategies in Biological Evolution. Ed. by L.H. Caporale. Annals of the New York Academy of Science. 870: 23-35. 
Shapiro, J.A. 2005. A 21st century view of evolution: genome system architecture, repetitive DNA, and natural genetic engineering. Gene 345: 91-100.

Simpson, G.G. 1953. The Major Features of Evolution. New York: Columbia University Press.

Simpson, G.G. 1984 [1944]. Tempo and Mode in Evolution. New York: Columbia University Press.

Sniegowski, P.D., Gerrish, P.J. and R.E. Lenski. 1997. Evolution of high mutation rates in experimental populations of E. coli. Nature 387: 703-705.

Sniegowski, P.D. and R.E. Lenski. 1995. Mutation and adaptation: The directed mutation controversy in evolutionary perspective. Annual Review of Ecology and Systematics 26: 553-578.

Stebbins, G.L. 1966. Processes of Organic Evolution. Englewood Cliffs: Prentice-Hall Inc.

Sternberg, R.V. 2002. On the roles of DNA repetitive elements in the context of a unified genomicepigenetic system. Annals of the New York Academy of Science 981: 154-188.

Taddei, F. 1995. Environnement et contrôles de la variabilité génétique chez Escherichia coli. Doctoral dissertation. University of Paris 11 Orsay.

Taddei, F., Vulic, M., Radman, M. and I. Matic. 1997. Genetic variability and adaptation to stress. In Environmental Stress Adaptation and Evolution. Ed. by R. Bijlsma and V. Loeschcke. Basel-New York: Birkhäuser Verlag.

Tenaillon, O., Le Nagard, H., Godelle, B. and F. Taddei. 2000. Mutators and sex in bacteria: conflict between adaptive strategies. Proceedings of the National Academy of Sciences USA 97: 10465-10470.

Tenaillon, O., Denamur, E. and I. Matic. 2004. Evolutionary significance of stress induced mutagenesis. Trends in Microbiology 12: 264-270.

Weber, M. 1996. Evolutionary plasticity in prokaryotes: a Panglossian view. Biology and Philosophy 11: 67-88.

Weismann, A. 1892 [1883]. Ueben Vererbung, (french translation) De l'Hérédité. In La découverte des lois de l'hérédité (1862-1900) : une anthologie. Ed. by C. Lenay. Presse Pocket (1990).

Weismann, A. 1893 [1892]. Das Keimplasma. Eine Theorie der Vererbung. Jena: Gustav Fischer. (english translation) The Germ Plasm. A Theory of Heredity. London: The Contemporary Science Series.

Wright, B.E., Longacre, A. and J.M. Reimers. 1999. Hypermutation in derepressed operons of Escherichia coli K12. Proceedings of the National Academy of Sciences USA 96: 5098-5094.

Wright, B.E. 2000. A biochemical mechanism for nonrandom mutations and evolution. Journal of Bacteriology 182: 2993-3001.

Wright, S. 1931. Evolution in Mendelian populations. Genetics 16: 97-159.

Zamenhof, S., Heldenmuth, L.H. and P.J. Zamenhof. 1966. Studies on mechanisms for the maintenance of constant mutability: mutability and the resistance to mutagens. Proceedings of the National Academy of Sciences USA 55: 50-58.

\section{Notes}

1. Biologists use both terms "chance" and "random" to describe the character of genetic mutations. I will usually restrict myself in this paper to the broader term "chance" to designate the consensus view on genetic mutations. In fact, the term "random" can be interpreted according to more specific mathematical connotations (Merlin 2009).

2. See Cairns and Foster 1991; Foster 2000, 2004; Hall 1998; Rosenberg 2001; Hastings et al. 2004; Shapiro 1999, 2005; Lenski and Mittler 1993; Miller 1996; Sniegowski et al. 1997; Moxon et al. 1994, 2006; Taddei et al. 1997; Tenaillon et al. 2000; Radman et al. 2000; Giraud et al. 2001; Caporale 1999, 2000, 2003; Wright et al. 1999, Wright 2000; Tenaillon et al. 2004; Galhardo et al. 2007.

3. Fisher 1999 [1930]; Wright 1931; Haldane 1966 [1932]; Dobzhansky 1951 [1937]; Mayr 1942; Huxley 1948 [1942]; Simpson 1984 [1944]; Simpson 1953; Huxley 1953; Rensch 1959; Mayr 1977 [1963]; Stebbins 1966; Dobzhansky et al. 1977. 
4. In Darwin's writings (e.g., see Darwin 1859, 131; 1868, 249; 1903, 312), we can distinguish different ways of understanding the notion of "chance variation" (Beatty 1984, 1987, 2006, 2008; Eble 1999; Lennox 2010). According to the most influential and persistent meaning in biology, variations are not caused because they can provide adaptation to the individual organisms concerned and their offspring. In this sense, variations are a matter of chance.

5. On the one hand, two events $\mathrm{A}$ and $\mathrm{B}$ are statistically (or probabilistically) independent if and only if $\operatorname{Pr}(\mathrm{A} \cap \mathrm{B})=$ $\operatorname{Pr}(\mathrm{A}) \times \operatorname{Pr}(\mathrm{B})$. On the other hand, two events $\mathrm{A}$ and $\mathrm{B}$ are causally independent if and only if $\mathrm{A}$ does not cause $\mathrm{B}$, $\mathrm{B}$ does not causes $\mathrm{A}$, and $\mathrm{A}$ and $\mathrm{B}$ do not have a common cause. The fact that two events $\mathrm{A}$ and $\mathrm{B}$ are statistically independent does not provide any evidence about their causal independence, and vice versa.

6. I use the expression "biologists of the Modern Synthesis" to designate both biologists usually considered as actors of the Modern Synthesis (1930s-1950s) - e.g., Dobzhansky, Simpson, Stebbins, Huxley, Mayr, Fisher, Haldane, Wright, Rensch - and biologists who did not participate to the Synthesis but agreed with its main assumptions at that time.

7. In particular, Zamenhof and his colleagues investigated the alteration of mutability in relation to environmental conditions (Zamenhof et al. 1966), and Leigh considered the adaptive evolution of mutation rate (Leigh 1973). It is also worth mentioning that some of biologists of the Modern Synthesis advanced the hypothesis of directed mutations, not from the point of view of their adaptive value, but as to the place they occur and their abundance (i.e., mutations that would occur in particular genomic sites with a specific probability; Dobzhansky 1951 [1937], 62-63). This hypothesis called into question the wholly random character of all genetic mutations (Mayr 1942, 68). Nevertheless, biologists of the Modern Synthesis were very cautious about this point and always pleaded ignorance of the physiology of the genes and of the chemistry of the mutational process.

8. As regards the connection between mutation rate and environmental factors, Dobzhansky claimed that "the genotype is neither unchangeable nor independent of the environment. Genotypic changes do occur in which the environment plays the role of at least a trigger mechanism [...]. Moreover, any genotype is the result of an agelong process of evolutionary development, in which the environment, through natural selection, has been a force of paramount importance. The structure of the genotype, and hence the kind of changes it is capable of producing, are in the last analysis environmentally determined. The 'determining environment' is, however, not the one prevailing at the moment, but rather it is the sum of the historical environments to which the organism had been exposed in its phylogeny" (1951 [1937], 21).

9. More precisely, Simpson claimed that a mutation in a poorly-adapted organism or living in a changing environment has greater chance to be advantageous than a mutation occurring in a well-adapted organism or situated in a stable environment. But this point is controversial and ignores the complexity of each particular situation. For Simpson, the adaptive value of a genetic mutation simply depends on the adaptedness of the organism concerned to its environment. However, it is also relative to other factors such as the kind of modification at the level of the nucleotide sequence, the genomic context where it occurs, and the effect of the genetic modification at the phenotypic level.

10. Millstein argues that "[...] a mutation is directed if and only if it is specifically caused by environmental stress in an exclusively adaptive manner" (Millstein 1997, 151).

11. The term "exclusively" must not be interpreted literally. Moreover, it is not essential to the definition of the notion of "evolutionary chance mutation." Millstein uses it to avoid some problems that arise in Sarkar's "weak" definition of "directed mutation" (Sarkar 1991). Sarkar claims that a mutation is directed "if it occurs (or occurs more frequently) in the fitness-enhancing or 'selective' environment", i.e., "in an environment where its associated phenotype has an enhanced fitness." The inherent risk of this definition is that it would consider a mutation to be "directed" even if it turns out to be beneficial in a given environment but not clearly more probable than other deleterious or neutral mutations. Sarkar's definition of "directed mutation" could also erroneously be applied to mutations which occur more frequently in a given environment when they are beneficial than in other environments where they are not, but not because of their adaptive value (in other terms, not because of the characteristics of the physico-chemical process causing them).

12. Millstein's definition of "directed mutation" - that I reformulate in the negative in order to define the notion of "evolutionary chance mutation" - is reminiscent of Lenski and Mittler's definition of "directed mutation" (Lenski and Mittler 1993, 188). They claim that a directed mutation is a mutation that "occurs at a higher rate specifically when (and even because) it is advantageous to the organism, whereas comparable increases in rate do not occur (i) in the same environment for similar mutations that are not advantageous, and (ii) for the same mutation in similar 
environments where it is not advantageous." Conditions (i) and (ii) suggested by Lenski and Mittler respectively correspond to the conditions (2) and (1) I have suggested above.

13. Luria and Delbrück's experiment is usually considered as providing the first evidence for "evolutionary chance" mutations, even though before, in 1934, Yang and White arrived to the same conclusion by observing the distribution of variants in colonies of $V$. cholerae (Sarkar 1991). For a detailed presentation and a critical discussion of Luria and Delbrück's fluctuation test, see Sarkar 1991; Sniegowski and Lenski 1995; Taddei 1995; Darden 2006; Merlin 2009.

14. Four years before, the result of Shapiro's experiments with E. coli bacteria had already suggested the possibility of "directed" mutations with respect to adaptation (Shapiro 1984).

15. Cairns and his colleagues even proposed some possible and apparently Lamarckian molecular mechanisms for "directed" mutations, for instance what they called "the specific reverse transcription" and "the non-specific reverse transcription" (Cairns et al. 1988; Cairns and Foster 1991).

16. For further details and bibliography about the "directed" mutation controversy see Sarkar 1991; Lenski and Mittler 1993; Jablonka and Lamb 1995; Sniegowski and Lenski 1995; Taddei 1995; Millstein 1997; Darden 2006; Merlin 2009.

17. Major advances in the study of mutator mechanisms have been made in microbiology research, in particular on some species of bacteria (Escherichia coli, Neisseria meningitidis, Staphylococcus aureus, Streptococcus pneumoniae, and Pseudomonas aeruginosa). There are two main reasons for this: first, growth rate of microorganisms is very high, therefore experiments can be realized in relatively short time; second, bacteria often live in changing environmental conditions, therefore they represent a particularly appropriate case-study for research on mutator mechanisms. My analysis is focused on mutations due to mutator mechanisms in microorganisms. Analogous mechanisms have been studied in other prokaryotes, and in unicellular and multi-cellular eukaryotes too. For examples and bibliography, see Jablonka and Lamb 1995.

18. The Methyl-Directed Mismatch Repair System in E. coli bacteria is an example of the inactivation of the repair system of DNA replication and, more generally, of a constitutive mutator mechanism provoking a global increase of mutation rate. Such a mechanism is usually present in stressful environmental conditions: it is due to a mutation in the genes of repair DNA polymerases (in E. coli bacteria, the mut genes) and provokes a permanent increase of mutation rate across the entire genome. If the mutator allele is selected because of its physical proximity (link) with a beneficial mutation occurred ("hitchhiking" or "second-order selection"), its frequency can increase in the population over time: once the mutator allele becomes fixed in the population, the mutator mechanism is considered as a mutational response to environmental challenges acquired by the population concerned (what biologists call a “constitutive mutator” population). See Radman \& Wagner 1986, Taddei et al. 1997.

19. "Hot-spots" hypermutability is due to the presence of some repeated nucleotide sequences - the "microsatellites" of two or three nucleotides, as ATATATAT - which can disturb the activity of the enzymes of replication (the DNA polymerases) by inducing them to slippage, and by provoking frame-shift mutations. For instance, mutational "hotspots" have been observed in genes involved in the production of cellular factors interacting with highly unpredictable environment (e.g., in Neisseria and Influenzae's pathogenic bacteria's genome amongst genes coding for surface proteins, which are very important in the interaction with host defenses; in antigens of the immune system of the host, which directly interact with pathogens). See Moxon et al. 1994, 2006.

20. The "SOS system", discovered by Radman (1973) in E. coli bacteria, consists of more than twenty genes whose expression is induced by the presence of DNA breakages or strong metabolic stresses (e.g., starvation). These genes code for inhibitor and activator protein factors (e.g., LexA and RecA in E. coli) and for special kinds of "error-prone lesion by pass" DNA-polymerases: some kinds of DNA-polymerases (e.g., Pol V in E. coli) can perform the so-called translesion-synthesis (TLS), i.e., can bypass the replication blocks caused by lesions, but have a reduced copying fidelity, so they produce localized mutations in the damaged regions; other kinds of DNA-polymerases (e.g., Pol IV in E. coli) can also act on undamaged DNA regions and so provoke genome-wide mutations. The result is a global and/or local increase of the mutation rate. Once the stress stimulus wears off, there is no more induction of the SOS genes and the mutation rate decreases to its normal average value. See Taddei 1995, Taddei et al. 1997, Tenaillon et al. 2004.

21. Weismann's quotation continues as follows: "Were it possible for growth to take place under absolutely constant external influences, variation would not occur; but as this is impossible, all growth is connected with smaller or greater deviations from the inherited developmental tendency. When these deviations only affect the soma, they 
give rise to temporary non-hereditary variations; but when they occur in the germ-plasm, they are transmitted to the next generation and cause corresponding hereditary variations in the body" (1893 [1892], 463; emphasis in the text).

22. For an argument in favor of selection for mutator mechanisms at a higher level than the individual (group selection), see Weber 1996.

\section{AGKNOWLEDGMents}

I would like to thank Jean Gayon, Michel Morange, Roberta Millstein, Frédéric Bouchard, Evelyn Fox Keller, Eric Bapteste, Daniel Parker, the people of the Institut d'Histoire et de Philosophie des Sciences et des Techniques (Paris), the TaMaRA Lab (Paris), and the anonymous referees for helpful comments and/or discussion; Lukas Sonderstrom for proofreading the most recent version of my paper; Brian Hill, Neil Kennedy, Vadim Keyser and Amanda Hodson for correcting earlier drafts; the Fonds Québecois de la Recherche sur la Nature et les Technologies for the financial support.

\section{Copyright (C) 2010 Author(s).}

This is an open-access article distributed under the terms of the Creative Commons Attribution-NonCommercial-NoDerivs license, which permits anyone to download, copy, distribute, or display the full text without asking for permission, provided that the creator(s) are given full credit, no derivative works are created, and the work is not used for commercial purposes.

ISSN 1949-0739 